\title{
DPYSL3 is a mutifunctional modulator in claudin-low breast cancer
}

\author{
Ryoichi Matsunuma ${ }^{1-3}$ and Matthew J Ellis ${ }^{1 *}$ \\ ${ }^{1}$ Lester and Sue Smith Breast Center, Baylor College of Medicine, Houston, USA \\ ${ }^{2}$ First Department of Surgery, Hamamatsu University School of Medicine, Hamamatsu, Japan \\ ${ }^{3}$ Hamamatsu Oncology Center, Japan
}

\section{Commentary}

Proteogenomics is the field of integrating data from mass spectrometry-based shotgun proteomics, and phosphoproteomics into next-generation RNA and DNA sequencing data analysis pipelines that promises new insights into cancer biology and therapeutic targeting. As well as analyses of clinical samples for disease phenotype association analysis, the application of proteogenomics to model systems also has considerable potential. Patient-derived xenografts (PDX) generated in immunosuppressed mice strains provide a useful setting to analyze the biological properties of the intrinsic subtypes of breast cancer because this approach effectively captures the biological diversity of this disease [1]. The Clinical Proteomic Tumor Analysis Consortium (CPTAC) generated quantitative i-TRAQ mass spectrometry-based proteomics and phosphoproteomics data across the WHIM series of PDXs tumors that was combined with RNA and DNA sequencing information to provide integrated proteogenomic profiles [2]. Herein we explored these data to identify extreme outliers in the proteogenomic data that were Claudin-low (CLOW) subtype-specific and had not previously studied in breast cancer. WHIM12 breast cancer PDX was previously classified as a high confidence CLOW tumor based on transcriptomic profiling [2]. A CPTAC proteogenomic analysis prioritized dihydropyrimidinaselike-3 (DPYSL3) as a multi-level (RNA/Protein/Phosphoprotein) expression outlier specific to the CLOW subset of triple negative breast cancers. These data suggested high-levels of DPYSL3 expression and hyper-phosphorylation were associated with CLOW breast cancer and thus DPYSL3 may regulate some of the unique biological features of this subtype. In our view, discovery approaches that trangulate multiple tiers of 'omics data with literature search engines to identify novel and targetable cancer biology should be more widely applied.

DPYSL3 is a phosphoprotein and a member of DPYSL gene family that shares about 50-70 \% sequence homology [3-6]. DPYSL3 is highly expressed in developing and adult nervous systems [6-8] and functions in a variety of cellular processes, including cell migration, differentiation, neurite extension, and axonal regeneration $[9,10]$. DPYSL3 regulates the actin cytoskeleton in neuroblastoma cells to inhibit cell migration [11]. DPYSL3 is also reported to play a role in cell migration and metastasis suppression in prostate cancer. However, in pancreatic cancer, DPYSL3 is positively associated with liver metastasis and poor outcome [12-16]. But DPYSL3 is understudied in breast cancer.

Breast cancer classification divided into four intrinsic subtypes based on gene expression profiling (Luminal A, Luminal B, HER2enriched, basal-like) which are associated with different patterns of recurrence and therapeutic responses $[17,18]$. Further gene expression studies have identified additional less common intrinsic subtypes, one of which was a CLOW subtype. The CLOW subtype indicates lack of expression from claudin family members that are components of tight junctions, where they form an intracellular barrier that regulates the flow of molecules between the cells of an epithelium. The incidence of CLOW tumors is reported to be between 7 to $14 \%$ of breast cancers and there is an association with metaplastic features whereby breast cancers show squamous or mesenchymal differentiation patterns $[19,20]$. The clinical significance of a diagnosis of a CLOW tumor is not established, mostly because there are few reports of a specific therapeutic vulnerability for this subtype, unlike other breast cancer subtypes.

In our study, DPYSL3 knock-down in DPYSL3 ${ }^{+}$CLOW cell lines demonstrated reduced proliferation, yet enhanced motility and increased expression of Epithelial-to-Mesenchymal Transition (EMT) markers suggesting that DPYSL3 is a multi-functional signaling modulator [21]. Slower proliferation in DPYSL $3^{\circledR}$ CLOW cells was associated with accumulation of multi-nucleated cells and polyploidy indicating a mitotic defect that was associated with a collapse of the vimentin microfilament network induced by vimentin hyperphosphorylation. On the other hand, DPYSL3 suppressed the expression of EMT regulators such as Snail and Twist and opposed p21 activated kinase 2 (PAK2) dependent migrations, However, these EMT regulators, in turn, induce DPYSL3 expression, suggesting DPYSL3 participates in negative feedback on EMT.

The role of DPYSL3 in preventing multiple nucleation and polyploidy during mitosis of CLOW cells was confirmed by studies in multiple cell lines. DPYSL3 interacts directly to tubulin, promotes microtubule assembly and is also known as a microtubule associated protein [22,23]. In fact, Immunoprecipitation and mass spectrometry data from CLOW cell line models demonstrates DPYSL3 interacts with tubulin $\beta$. In addition, there are some previous reports that demonstrate DPYSL3 regulates the actin and microtubule growth cone cytoskeleton $[10,11,22,24]$, and other publications demonstrate GSK3 regulates mitotic chromosomal alignment through phosphorylation of DPYSL3 and mitotic progression through its effect on spindle microtubules [25]. In CLOW breast cancer, loss of DPYSL3 causes multiple nucleation and

${ }^{*}$ Correspondence to: Matthew J. Ellis, Lester and Sue Smith Breast Center, Baylor College of Medicine, Houston, Texas 77030, USA, Tel: (713) 798-1999; E-mail: matthew.ellis@bcm.edu

Received: November 22, 2018; Accepted: December 10, 2018; Published: December 14, 2018 
then polyploidy due to cytokinetic failure likely due to disruption of vimentin function through increased phosphorylation. In other words DPYSL3 is a negative regulator of a vimentin protein kinase(s) which has yet to be identified.

In contrast, the role of DPYSL3 in migration and EMT in CLOW cells does appear to be PAK dependent and specifically mediated by PAK2. Cell migration in DPYSL3- cells correlated with increased phosphorylation of PAK2 on Ser20 and was sensitive to PAK2 siRNA and pharmacological PAK inhibition. Immunoprecipitation and mass spectrometry-based proteomics or western blotting suggested that DPYSL3 and PAK2 directly interact such that DPYSL3 may function as a direct negative regulator of PAK2. Thus a PAK inhibitor could potentially mitigate increase migration as an adverse effect of DPYSL3 targeting. Importantly a PAK inhibitor does not differentially affect the viability of WHIM12 shLuc versus WHIM12 shDPYSL3 cells and a PAK inhibitor had no effect on a distribution of cell cycle in both of WHIM12 shLuc and shDPYSL3. Thus, DPYSL3 can be considered a biomarker for CLOW tumors where migration and EMT can be potentially resistant to a PAK inhibitor.

A role for DPYSL3 in EMT is consistent with Parsana's multistudy integration data [26]. Furthermore WHIM12 was derived from a patient with a metaplastic carcinoma with spindle cell formation, an EMT characteristic. Further analyses of human data (METABRIC) in tumors designated CLOW subtype revealed a strong positive correlation between multiple EMT markers and DPYSL3 expression. This finding is consistent with induction of DPYSL3 by Snail and Twist in the HMLE EMT system in which HMLE-Snail and HMLE-Twist cells were generated by infecting immortalized human mammary epithelial cells with retroviral vectors expressing Snail or Twist. While it appears somewhat paradoxical that a negative regulator of EMT is positively associated with EMT markers in clinical data sets a logical synthesis of these data is that DPYSL3 is induced by EMT and subsequently provides negative feedback on the EMT state by inhibiting PAK. Within a tumor where EMT is the dominant and pathological state, DPYSL3 mediated feedback maybe impaired; causing DPYSL3 levels to increase yet EMT persists. DPYSL3 is induced by retroviral expression of Twist and Snail in this model, thus potential feedback inhibition in the transcription of Snail and Twist by DPYSL3 is disrupted because these EMT transcription factors are not controlled by their normal regulatory elements. Certainly the kinetics and exact mechanism of how DPYSL3 regulates EMT warrants further detailed study.

In conclusion, our study provides insights into DPYSL3 as a negative regulator of kinases that modulate cytokinesis, motility and EMT in CLOW breast cancer. DPYSL3 expression identifies CLOW tumors that will be sensitive to approaches that promote vimentin phosphorylation during mitosis and inhibitors of PAK signaling during migration and EMT. Therefore DPYSL3 expression could thus be used to stratify patients for clinical trials that target these cancer hallmarks.

\section{References}

1. Li S, Shen D, Shao J, Crowder R, Liu W, et al. (2013) Endocrine-Therapy-Resistant ESR1 Variants Revealed by Genomic Characterization of Breast-Cancer-Derived Xenografts. Cell Rep 4: 1116-1130. [Crossref]

2. Huang KL, Li S, Mertins P, Cao S, Gunawardena HP, et al. (2017) Proteogenomic integration reveals therapeutic targets in breast cancer xenografts. Nat Commun 8: 14864. [Crossref]

3. Goshima Y, Nakamura F, Strittmatter P, Strittmatter SM (1995) Collapsin-induced growth cone collapse mediated by an intracellular protein related to UNC-33. Nature 376: 509-514. [Crossref]
4. Gaetano C, Matsuo T, Thiele CJ (1997) Identification and characterization of a retinoic acid-regulated human homologue of the unc-33-like phosphoprotein gene (hUlip) from neuroblastoma cells. J Biol Chem 272: 12195-12201. [Crossref]

5. Quach TT, Mosinger B Jr, Ricard D, Copeland NG, Gilbert DJ, et al. (2000) Collapsin response mediator protein-3/unc-33-like protein-4 gene: organization, chromosoma mapping and expression in the developing mouse brain. Gene 242: 175-182. [Crossref]

6. Fukada M, Watakabe I, Yuasa-Kawada J, Kawachi H, Kuroiwa A, et al. (2000) Molecular characterization of CRMP5, a novel member of the collapsin response mediator protein family. J Biol Chem 275: 37957-37965. [Crossref]

7. Minturn JE, Fryer HJ, Geschwind DH, Hockfield S (1995) TOAD-64, a gene expressed early in neuronal differentiation in the rat, is related to unc-33, a C. elegans gene involved in axon outgrowth. J Neurosci 15: 6757-6766. [Crossref]

8. Yuasa-Kawada J, Suzuki R, Kano F, Ohkawara T, Murata M, et al. (2003) Axona morphogenesis controlled by antagonistic roles of two CRMP subtypes in microtubule organization. Eur J Neurosci 17: 2329-2343. [Crossref]

9. Charrier E, Reibel S, Rogemond V, Aguera M, Thomasset N, et al. (2003) Collapsin response mediator proteins (CRMPs): involvement in nervous system development and adult neurodegenerative disorders. Mol Neurobiol 28: 51-64. [Crossref]

10. Alabed YZ, Pool M, Ong Tone S, Fournier AE (2007) Identification of CRMP4 as a convergent regulator of axon outgrowth inhibition. J Neurosci 27: 1702-1711. [Crossref]

11. Rosslenbroich V, Dai L, Baader SL, Noegel AA, Gieselmann V, et al. (2005) Collapsin response mediator protein-4 regulates F-actin bundling. Exp Cell Res 310: 434-444. [Crossref]

12. Gao X, Pang J, Li LY, Liu WP, Di JM, et al. (2010) Expression profiling identifies new function of collapsin response mediator protein 4 as a metastasis-suppressor in prostate cancer. Oncogene 29: 4555-4566. [Crossref]

13. Li K, Pang J, Cheng H, Liu WP, Xiao HJ, et al. (2015) Manipulation of prostate cancer metastasis by locus-specific modification of the CRMP4 promoter region using chimeric TALE DNA methyltransferase and demethylase. Oncotarget 6: 10030-10044. [Crossref]

14. Zhou W, Xie P, Pang M, Yang B, Fang Y, et al. (2015) Upregulation of CRMP4, a new prostate cancer metastasis suppressor gene, inhibits tumor growth in a nude mouse intratibial injection model. Int J Oncol 46: 290-298. [Crossref]

15. Hiroshima Y, Nakamura F, Miyamoto H, Mori R, Taniguchi K, et al. (2013) Collapsin response mediator protein 4 expression is associated with liver metastasis and poor survival in pancreatic cancer. Ann Surg Oncol 20: S369-S378. [Crossref]

16. Li C, Jiang W, Hu W, Li LC, Dong L, et al. (2016) Enhancing DPYSL3 gene expression via a promoter-targeted small activating RNA approach suppresses cancer cell motility and metastasis. Oncotarget 7: 22893-22910. [Crossref]

17. Perou CM, Sørlie T, Eisen MB, van de Rijn M, Jeffrey SS, et al. (2000) Molecular portraits of human breast tumours. Nature 406: 747-752. [Crossref]

18. Sørlie T, Perou CM, Tibshirani R, Aas T, Geisler S, et al. (2001) Gene expression patterns of breast carcinomas distinguish tumor subclasses with clinical implications. Proc Natl Acad Sci U S A 98: 10869-10874. [Crossref]

19. Prat A, Parker JS, Karginova O, Fan C, Livasy C, et al. (2010) Phenotypic and molecular characterization of the claudin-low intrinsic subtype of breast cancer. Breast Cancer Res 12: R68-R68.

20. Weigelt B, Ng CK, Shen R, Popova T, Schizas M, et al. (2015) Metaplastic breast carcinomas display genomic and transcriptomic heterogeneity [corrected]. Mod Pathol 28: 340-351. [Crossref]

21. Matsunuma R, Chan DW, Kim BJ, Singh P, Han A, et al. (2018) DPYSL3 modulates mitosis, migration, and epithelial-to-mesenchymal transition in claudin-low breast cancer. Proc Natl Acad Sci U S A 115: E11978-E11987.

22. Khazaei MR, Girouard MP, Alchini R, Ong Tone S, Shimada T, et al. (2014) Collapsin Response Mediator Protein 4 Regulates Growth Cone Dynamics through the Actin and Microtubule Cytoskeleton. J Biol Chem 289: 30133-30143. [Crossref]

23. Lin PC, Chan PM, Hall C, Manser E (2011) Collapsin response mediator protein (CRMPs) are a new class of microtubule-associated protein (MAP) that selectively interacts with assembled microtubules via a taxol-sensitive binding interaction. J Biol Chem 286: 41466-41478. [Crossref]

24. Tan M, Cha C, Ye Y, Zhang J, Li S, et al. (2015) CRMP4 and CRMP2 Interact to Coordinate Cytoskeleton Dynamics, Regulating Growth Cone Development and Axon Elongation. Neural Plasticity 2015: 947423. 
25. Ong Tone S1, Dayanandan B, Fournier AE, Mandato CA (2010) GSK3 regulates mitotic chromosomal alignment through CRMP4. PLoS One 5: e14345. [Crossref]
26. Parsana P, Amend SR, Hernandez J, Pienta KJ, Battle A (2017) Identifying global expression patterns and key regulators in epithelial to mesenchymal transition through multi-study integration. BMC Cancer 17: 447.

Copyright: $\bigcirc 2018$ Matsunuma R. This is an open-access article distributed under the terms of the Creative Commons Attribution License, which permits unrestricted use, distribution, and reproduction in any medium, provided the original author and source are credited. 